\title{
Transmit/Receive Beamforming in Multiuser AF Relay Networks with Opportunistic Scheduling
}

\author{
Daniel Benevides da Costa, André L. F. de Almeida, and C. Alexandre R. Fernandes
}

\begin{abstract}
Resumo-Este artigo investiga o desempenho de outage de redes multiusuário com relays onde o terminal fonte e cada um dos nós destinos são equipados com múltiplas antenas e empregam técnicas de beamforming. Mais especificamente, assumindo canais de desvanecimento Rayleigh e um relay amplifica-e-emcaminha, uma expressão geral para a probabilidade de outage é obtida. Nossa análise leva em consideração os benefícios inerentes à seleção oportunística em redes assistidas por relay, no qual a fonte seleciona o terminal destino com o melhor canal para a transmissão de dados. Os resultados analíticos são validados por simulação Monte Carlo, e os efeitos da posição do relay e configuração de antenas no desempenho do sistema são discutidos através de alguns resultados numéricos.
\end{abstract}

Palavras-Chave-Técnicas de beamforming, comunicações cooperativas, diversidade multiusuário, seleção oportunística, probabilidade de outage.

Abstract-This paper evaluates the outage performance of multiuser relay networks in which the source and each one of the destinations are equipped with multiple antennas and employ beamforming techniques. More specifically, assuming Rayleigh fading channels and an amplify-and-forward relay, a general closed-form expression for the end-to-end outage probability is derived. Our analysis takes into account the benefits conferred by opportunistic scheduling in relay-assisted networks, where the source selects the destination with the strongest channel to schedule data transmission. The analytical results are validated by means of Monte Carlo simulation, and the effects of the relay placement and antenna configurations, on the overall system performance, are discussed through some representative numerical results and comparisons.

Keywords-Beamforming techniques, cooperative communications, multiuser diversity, opportunistic scheduling, outage probability.

\section{INTRODUCTION}

Along the last years, cooperative diversity has proven to be an efficient technique for meeting the continuous necessity of increased data rates and higher throughput inherent to wireless communications systems [1]-[4]. By allowing several mobile users (or relays) to share their antennas and resources, an antenna array is emulated so that the benefits of spatial diversity and/or spatial multiplexing can be exploited in a distributed manner. The ultimate goal is to extend coverage without using high power levels at the transmitter, hence increasing connectivity and capacity. Depending on the relay

D. B. da Costa and C. A. R. Fernandes are with the Federal University of Ceará - Campus Sobral, CE, Brazil (email: danielbcosta@ieee.org, alexandrefernandes@ufc.br). The work of D. B. da Costa is supported in part by the Banco do Nordeste do Brasil (BNB) and by the CNPq (Proc. 302106/2011-1). The work of C. A. R. Fernandes is supported in part by the Banco do Nordeste do Brasil (BNB) and by the FUNCAP (Proc. BPI-0031-00106.01.00/10).

A. L. F. de Almeida is with the Department of Teleinformatics Engineering, Federal University of Ceará, Fortaleza, Brazil (email: andre@gtel.ufc.br). This work was supported in part by the CNPq (Proc. 303238/2010-0). complexity, several cooperative strategies have been proposed in the literature, such as amplify-and-forward (AF), decodeand-forward (DF), and coded cooperation strategies. Among these, the AF mode is particularly attractive since it requires no decoding at the relay nodes, which results in a low complexity when compared to the other modes. In AF, the relay merely amplifies the signal received from the source and forwards its amplified version to the destination.

Recently, multiuser diversity (MUD), a kind of diversity inherent to multiuser systems [5], has attracted significant attention in cooperative communications. MUD exploits the fact that different users may undergo independent varying channels, and at any time slot, there may be a user whose channel gain is near to the peak. Then, the time-varying nature of the fading channel can be exploited in a more efficient way. Indeed, this kind of diversity can be seen as an analogy of the water-filling principle, where more resources are allocated to the user with better channel quality [6].

Currently, many papers have addressed the analysis of MUD in cooperative networks [7]-[12]. However, despite the practical importance, the deployment of beamforming techniques [13] in multiuser relay networks (MRNs) has never been investigated in the literature up to now. For example, the benefits acquired in [12] can be further improved by inserting beamforming techniques in the system model under consideration. Therefore, in order to partly fill this gap, this paper analyzes the outage performance of MRNs in which the source and each one of the destinations are equipped with multiple antennas and employ beamforming techniques. More specifically, assuming Rayleigh fading and a fixed-gain relay, a general closed-form expression for the end-to-end outage probability is derived. Our analysis takes into account the benefits conferred by opportunistic scheduling in relay-assisted networks. The analytical results are validated by means of Monte Carlo simulation, and the effects of relay placement and antenna configurations, on the overall system performance, are discussed through some representative numerical results and comparisons.

The remainder of this paper is organized as follows. In Section II, the system and channel models are introduced. In addition, the opportunistic scheduling algorithm is briefly described. In Section III, the outage probability (OP) is investigated. Specifically, a general closed-form expression for the outage probability of the considered system is derived. Numerical results and discussions are provided in Section IV. Concluding remarks are given in Section $\mathrm{V}$ and Appendix provides a detailed proof for an analytical expression derived in Section III.

Mathematical Notations and Functions: Vectors are shown 


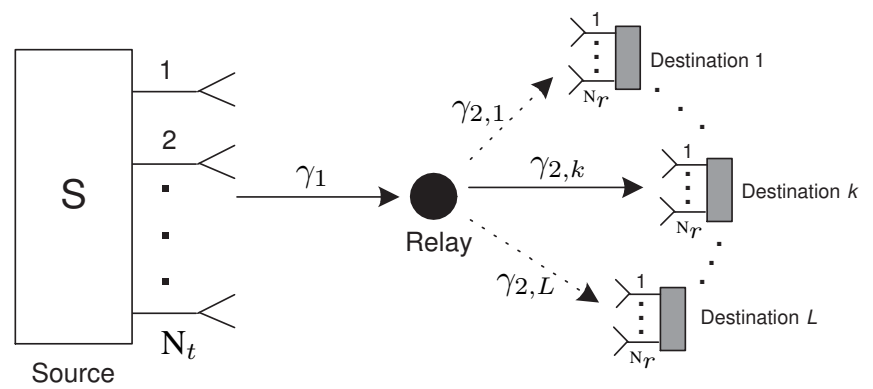

Fig. 1. Dual-hop multiuser cooperative relay network consisting of one source node with $\mathrm{N}_{t}$ antennas, one AF relay with a single antenna, and $L$ destinations with $\mathrm{N}_{r}$ antennas each.

with bold letters. $\mathrm{E}[\cdot]$ denotes expectation, $\|\cdot\|_{F}$ is the Frobenius norm, $(\cdot)^{\dagger}$ is the conjugate transpose operator, $\operatorname{Pr}[\cdot]$ symbolizes probability, $f_{X}(\cdot)$ and $F_{X}(\cdot)$ represent the probability density function (PDF) and cumulative distribution function (CDF) of the random variable $X$, respectively. $\Gamma(\cdot)$ and $\Gamma(\cdot, \cdot)$ indicate the Gamma function [14, Eq. 8.310.1] and the incomplete Gamma function [14, Eq. 8.350.2], respectively. $K_{x}(\cdot)$ denotes the $x$-order modified Bessel function of second kind [15, Eq. 9.6.22].

\section{System AND Channel Models}

We consider a system model similar to [12], i.e., one source $S$ communicates with one out of $L$ destinations by using a half-duplex AF relay $\mathcal{R}$ and an opportunistic scheduling algorithm is employed for selecting the best destination (i.e., the one with the highest end-to-end signal-to-noise ratio (SNR)). The basic difference between our system model and [12] is that here the source and each one of the $L$ destinations are equipped with $\mathrm{N}_{t}$ and $\mathrm{N}_{r}$ antennas, respectively, as shown in Fig. 1. In this case, in order to maximize the end-to-end SNR, the source and each one of the destinations employ transmit and receive beamforming ${ }^{1}$. For sake of clarity, in the sequel we briefly describe how the communication process is performed.

In the first time slot, the signal $x$ to be transmitted by the source is weighted with a $\mathrm{N}_{t} \times 1$ transmit beamforming vector $\mathbf{w}_{t}$ to form the transmitted signal vector ${ }^{2}$. Assuming that $x$ has an average power normalized to unity and incorporating the path loss in the signal propagation, the received scalar signal $y_{\mathcal{R}}$ at the relay can be written as

$$
y_{\mathcal{R}}=\sqrt{E_{S \mathcal{R}} d_{S \mathcal{R}}^{-\eta}} \mathbf{h}_{1} \mathbf{w}_{t} x+n_{1}
$$

where $\mathbf{h}_{1}$ is the $\mathrm{N}_{t} \times 1$ channel vector between the source and relay with Rayleigh fading entries, $E_{S \mathcal{R}}$ denotes the average symbol energy at the source, $d_{S \mathcal{R}}$ is the distance between the source and the relay, $\eta$ is the path loss exponent ${ }^{3}$, and $n_{1}$ is

\footnotetext{
${ }^{1}$ Please, check [13] for further details about beamforming techniques.

${ }^{2} \mathrm{We}$ assume that the channel state information (CSI) is available at the source.

${ }^{3}$ For outdoor propagation, $\eta$ ranges between 2 and 5, depending upon the radio environment. For instance, in free space, $\eta$ is equal to 2 , and when obstructions are present, $\eta$ has a larger value. In Section IV, our results will concentrate on a practical example of a highly shadowed urban area in which $\eta=4$.
}

the additive white Gaussian noise (AWGN) signal with singlesided power spectral $N_{0}$. As shown in [16], the optimum weight $\mathbf{w}_{t}$ that maximizes the received SNR at the relay is given by $\mathbf{w}_{t}=\frac{\mathbf{h}_{1}^{\dagger}}{\left\|\mathbf{h}_{1}\right\|_{F}}$. Defining $G$ as the scaling gain applied at the relay, we have that in the second time slot the received signal at the $k$ th destination from the relay is expressed as

$$
\begin{aligned}
y_{k}=\sqrt{E_{\mathcal{R} D} d_{\mathcal{R} D}^{-\eta}} \mathbf{w}_{r, k} \mathbf{h}_{2, k} G & \left(\sqrt{E_{S \mathcal{R}} d_{S \mathcal{R}}^{-\eta}} \mathbf{h}_{1}^{\dagger} \mathbf{w}_{t} x+n_{1}\right) \\
& +\mathbf{w}_{r, k} \mathbf{n}_{2, k},
\end{aligned}
$$

where $\mathbf{w}_{r, k}=\frac{\mathbf{h}_{2, k}^{\dagger}}{\left\|\mathbf{h}_{2, k}\right\|_{F}}, \mathbf{h}_{2, k}$ stands for the $\mathrm{N}_{r} \times 1$ channel vector between the relay and the $k$ th destination with Rayleigh fading entries, $E_{\mathcal{R} D}$ denotes the average symbol energy at the relay, $\mathbf{n}_{2, k}$ designates the $\mathrm{N}_{r} \times 1$ noise vector with singlesided power spectral $N_{0}$ at the $k$ th destination, and $d_{\mathcal{R} D}$ is the distance between the relay and the $k$ th destination. Here we assume that all destinations are equidistant from the relay, and hence $d_{\mathcal{R} D}$ is the same for all destinations.

By performing some algebraic manipulations in (2), the equivalent instantaneous end-to-end signal-to-noise ratio (SNR) at the $k$ th destination can be expressed as [17]

$$
\gamma_{\mathrm{end}, k}=\frac{\gamma_{1} \gamma_{2, k}}{\gamma_{2, k}+\frac{1}{G^{2} N_{0}}}
$$

where $\gamma_{1}=\frac{\left\|\mathbf{h}_{1}\right\|_{F}^{2} d_{S \mathcal{R}}^{-\eta} E_{S \mathcal{R}}}{N_{0}}$ and $\gamma_{2, k}=\frac{\left\|\mathbf{h}_{2, k}\right\|_{F}^{2} d_{\mathcal{R} D}^{-\eta} E_{\mathcal{R} D}}{N_{0}}$ denote the instantaneous SNRs of the first and second hops, respectively. Correspondingly, the per-hop average SNR is given by $\bar{\gamma}_{1}=\mathrm{E}\left[\gamma_{1}\right]$ and $\bar{\gamma}_{2, k}=\mathrm{E}\left[\gamma_{2, k}\right]$. As in [12], we assume that all the destinations are located in a homogeneous environment so that the signals from the relay to any antenna of the $L$ destinations experience independent and identically distributed (i.i.d.) Rayleigh fading. This implies that $\bar{\gamma}_{2, k}=\bar{\gamma}_{2}$. However, it is noteworthy that the first and second hops experience different per-hop average SNRs, i.e., $\bar{\gamma}_{1} \neq \bar{\gamma}_{2}$.

Note from (3) that the choice of the relay gain affects the determination of the instantaneous end-to-end SNR. In this case, when fixed-gain relays are employed, the gain $G$ established in the connection can be given by $G^{2}=1 /\left(C N_{0}\right)$, where $C$ is a constant. More specifically, assuming semi-blind relays, it can be easily shown that $C=\bar{\gamma}_{1}+1$ [12]. It should be emphasized that semi-blind relaying systems alleviates the task of continuous monitoring of the channel for its instantaneous knowledge, as in CSI-assisted relaying schemes, while offering a comparable performance to CSI-assisted schemes. Plugging the gain's expression in (3), it follows that

$$
\gamma_{\mathrm{end}, k}=\frac{\gamma_{1} \gamma_{2, k}}{\gamma_{2, k}+C}
$$

The PDFs of $\gamma_{1}$ and $\gamma_{2, k}$ can be written as

$$
f_{\gamma}(\gamma)=\frac{\gamma^{\mathrm{N}-1}}{\Gamma(\mathrm{N}) \bar{\gamma}^{\mathrm{N}}} \exp \left(-\frac{\gamma}{\bar{\gamma}}\right),
$$

where: (a) when $\gamma=\gamma_{1}$, we have $\bar{\gamma}=\bar{\gamma}_{1}$ and $\mathrm{N}=\mathrm{N}_{t}$, and (b) when $\gamma=\gamma_{2, k}$, we have $\bar{\gamma}=\bar{\gamma}_{2}$ and $\mathrm{N}=\mathrm{N}_{r}$.

In order to decide which destination will be served by the source in a given transmission process, an opportunistic scheduling algorithm is employed. Then, the destination with 
the highest instantaneous end-to-end SNR, denoted as $\gamma_{\mathrm{end}}$, can be determined as

$$
\gamma_{\mathrm{end}}=\max _{1 \leq k \leq L}\left\{\gamma_{\mathrm{end}, k}\right\}
$$

Such a algorithm is implemented at the relay. To make it work properly, we assume that the relay has perfect knowledge of the relay-destination links that are scheduled for transmission. In this case, at the beginning of each scheduling period, the relay broadcasts a destination synchronization message (DSM), which contains the synchronization information and the pilot signaling transmission order ${ }^{4}$. Each destination conveys pilot signals to the relay according to the transmission order contained in the DSM. At the relay, channel estimation is performed based on a pilot signal sequence sent by the $L$ destinations. The relay identifies and chooses the strongest destination and then feeds back the index of the strongest destination to the source.

It should be emphasized that, at each selection period, a short guard interval is introduced in order to allow the relay perform the channel estimations of the relay-destination links and make the required comparisons for destination's selection. Also, with the aim to avoid continuous selection process, it is assumed that the relay updates the selection only during the guard interval before each data burst and that the length of the data burst is approximately the channel coherence time.

\section{OUtage Probability Analysis}

An important performance measure in wireless systems is the outage probability. In MRNs with opportunistic scheduling, the OP is defined as the probability that $\gamma_{\text {end }}$ falls below a predefined threshold $\gamma_{\text {th }}$. This threshold is a protection value for the SNR, above which the quality-of-service is deemed satisfactory. Such a metric can be mathematically formulated as

$$
P_{\text {out }}=\operatorname{Pr}\left[\gamma_{\text {end }}<\gamma_{\text {th }}\right]=F_{\gamma_{\text {end }}}\left(\gamma_{\mathrm{th}}\right),
$$

which is indeed the CDF of $\gamma_{\text {end }}$ evaluated at $\gamma_{\text {th }}$. In what follows, a closed-form expression will be derived for the outage probability.

Knowing that the power gains of the $L$ relay-destination links undergo i.i.d. Gamma distributions, this metric can be expressed as [12]

$$
\begin{aligned}
F_{\gamma_{\text {end }}}\left(\gamma_{\text {th }}\right) & =\int_{0}^{\infty} \operatorname{Pr}\left[\gamma_{\text {end }, k}<\gamma_{\mathrm{th}} \mid \gamma_{1}\right]^{L} f_{\gamma_{1}}\left(\gamma_{1}\right) d \gamma_{1} \\
& =\int_{0}^{\infty} \operatorname{Pr}\left[\frac{\gamma_{1} \gamma_{2, k}}{\gamma_{2, k}+C}<\gamma_{\mathrm{th}} \mid \gamma_{1}\right]^{L} f_{\gamma_{1}}\left(\gamma_{1}\right) d \gamma_{1} \\
& =\int_{0}^{\gamma_{\mathrm{th}}} \operatorname{Pr}\left[\gamma_{2, k}>\frac{C \gamma_{\mathrm{th}}}{\gamma_{1}-\gamma_{\mathrm{th}}} \mid \gamma_{1}\right]^{L} f_{\gamma_{1}}\left(\gamma_{1}\right) d \gamma_{1} \\
& +\int_{\gamma_{\mathrm{th}}}^{\infty} \operatorname{Pr}\left[\gamma_{2, k}<\frac{C \gamma_{\mathrm{th}}}{\gamma_{1}-\gamma_{\mathrm{th}}} \mid \gamma_{1}\right]^{L} f_{\gamma_{1}}\left(\gamma_{1}\right) d \gamma_{1} \\
& =I_{1}+I_{2} .
\end{aligned}
$$

\footnotetext{
${ }^{4}$ It is assumed that the destinations are perfectly synchronized to the relay
} in time. This is a feasible assumption in TDMA cellular networks [18].
Knowing that $\operatorname{Pr}\left[\gamma_{2, k}>\left(C \gamma_{\text {th }}\right) /\left(\gamma_{1}-\gamma_{\text {th }}\right) \mid \gamma_{1}\right]=1$ for $0<$ $\gamma_{1}<\gamma_{\text {th }}, I_{1}$ can be easily calculated as

$$
I_{1}=\int_{0}^{\gamma_{\mathrm{th}}} f_{\gamma_{1}}\left(\gamma_{1}\right) d \gamma_{1}=1-\frac{\Gamma\left(\mathrm{N}_{t}, \frac{\gamma_{\mathrm{th}}}{\bar{\gamma}_{1}}\right)}{\Gamma\left(\mathrm{N}_{t}\right)}
$$

Note that (9) represents the CDF of $\gamma_{1}$. Based on [14, Eq. (8.352.2)], such CDF can be rewritten so that

$$
I_{1}=1-\exp \left(-\frac{\gamma_{\mathrm{th}}}{\bar{\gamma}_{1}}\right) \sum_{p=0}^{\mathrm{N}_{t}-1} \frac{\gamma_{\mathrm{th}}^{p}}{p ! \bar{\gamma}_{1}^{p}} .
$$

Turning our attention for the calculation of $I_{2}$, this term can be obtained as

$$
I_{2}=\int_{\gamma_{\text {th }}}^{\infty} \underbrace{\left[1-\frac{\Gamma\left(\mathrm{N}_{r}, \frac{C \gamma_{\text {th }}}{\bar{\gamma}_{2}\left(\gamma_{1}-\gamma_{\text {th }}\right)}\right)}{\Gamma\left(\mathrm{N}_{r}\right)}\right]^{L}}_{\Xi} \frac{\gamma_{1}^{\mathrm{N}_{t}-1}}{\Gamma\left(\mathrm{N}_{t}\right) \bar{\gamma}_{1}^{\mathrm{N}_{t}}} e^{-\frac{\gamma_{1}}{\bar{\gamma}_{1}}} d \gamma_{1} .
$$

In Appendix, it is shown that $\Xi$ is given by

$$
\Xi=\sum_{k=0}^{L}\left(\begin{array}{l}
L \\
k
\end{array}\right)(-1)^{k} \exp \left(-\frac{C \gamma_{\mathrm{th}} k}{\bar{\gamma}_{2}\left(\gamma_{1}-\gamma_{\mathrm{th}}\right)}\right) \sum_{l=0}^{k\left(\mathrm{~N}_{r}-1\right)} \beta_{l}^{k} \varphi^{l}
$$

where $\varphi=C \gamma_{\text {th }} /\left(\bar{\gamma}_{2}\left(\gamma_{1}-\gamma_{\text {th }}\right)\right)$ and

$$
\beta_{0}^{k}=1, \beta_{1}^{k}=k, \beta_{k\left(\mathrm{~N}_{r}-1\right)}^{k}=\left(\frac{1}{\left(\mathrm{~N}_{r}-1\right) !}\right)^{k},
$$

and

$$
\beta_{l}^{k}=\frac{1}{l} \sum_{v=1}^{p} \frac{v(k+1)-l}{v !} \beta_{l-v}^{k},
$$

with $p=\min \left(l, \mathrm{~N}_{r}-1\right), 2 \leq l \leq k\left(\mathrm{~N}_{r}-1\right)-1$. Вy substituting (12) into (11), $I_{2}$ can be evaluated as

$$
\begin{aligned}
& I_{2}=\frac{1}{\Gamma\left(\mathrm{N}_{t}\right) \bar{\gamma}_{1}^{\mathrm{N}_{t}}} \sum_{k=0}^{L}\left(\begin{array}{l}
L \\
k
\end{array}\right)(-1)^{k} \sum_{l=0}^{k\left(\mathrm{~N}_{r}-1\right)} \beta_{l}^{k} \int_{\gamma_{\mathrm{th}}}^{\infty} e^{-\frac{\gamma_{1}}{\gamma_{1}}} \\
& \times\left(\frac{C \gamma_{\mathrm{th}}}{\bar{\gamma}_{2}\left(\gamma_{1}-\gamma_{\mathrm{th}}\right)}\right)^{l} e^{-\frac{C \gamma_{\mathrm{th}} k}{\bar{\gamma}_{2}\left(\gamma_{1}-\gamma_{\mathrm{th}}\right)}} \gamma_{1}^{\mathrm{N}_{t}-1} d \gamma_{1} \\
& =\frac{1}{\Gamma\left(\mathrm{N}_{t}\right) \bar{\gamma}_{1}^{\mathrm{N}_{t}}} \sum_{k=0}^{L}\left(\begin{array}{l}
L \\
k
\end{array}\right)(-1)^{k} \sum_{l=0}^{k\left(\mathrm{~N}_{r}-1\right)} \beta_{l}^{k} I_{3} .
\end{aligned}
$$

Now, by performing the change of variables $\lambda=\gamma_{1}-\gamma_{\mathrm{th}}$, the term $I_{3}$ in (15) can be reexpressed as

$$
I_{3}=e^{-\frac{\gamma_{\mathrm{th}}}{\bar{\gamma}_{1}}} \int_{0}^{\infty}\left(\frac{C \gamma_{\mathrm{th}}}{\bar{\gamma}_{2} \lambda}\right)^{l} e^{-\frac{C \gamma_{\mathrm{th}} k}{\bar{\gamma}_{2} \lambda}-\frac{\lambda}{\bar{\gamma}_{1}}}\left(\lambda+\gamma_{\mathrm{th}}\right)^{\mathrm{N}_{t}-1} d \lambda
$$

Expressing $\left(\lambda+\gamma_{\mathrm{th}}\right)^{\mathrm{N}_{t}-1}$ in terms of finite sums (using the binomial theorem), (16) can be rewritten as

$$
\begin{aligned}
I_{3}=e^{-\frac{\gamma_{\mathrm{th}}}{\bar{\gamma}_{1}}}\left(\frac{C \gamma_{\mathrm{th}}}{\bar{\gamma}_{2}}\right)^{l} & \sum_{x=0}^{\mathrm{N}_{t}-1}\left(\begin{array}{c}
\mathrm{N}_{t}-1 \\
x
\end{array}\right) \gamma_{\mathrm{th}}^{\mathrm{N}_{t}-1-x} \int_{0}^{\infty} \lambda^{x-l} \\
& \times e^{-\frac{C \gamma_{\mathrm{th}} k}{\bar{\gamma}_{2} \lambda}-\frac{\lambda}{\bar{\gamma}_{1}}} d \lambda
\end{aligned}
$$


In order to solve (17), we make use of the identity given in [14, Eq. (3.324.1)], which results in

$$
\begin{aligned}
I_{3} & =2 \exp \left(-\frac{\gamma_{\mathrm{th}}}{\bar{\gamma}_{1}}\right)\left(\frac{C}{\bar{\gamma}_{2}}\right)^{l} \sum_{x=0}^{\mathrm{N}_{t}-1}\left(\begin{array}{c}
\mathrm{N}_{t}-1 \\
x
\end{array}\right) \gamma_{\mathrm{th}}^{\frac{1}{2}(l-1-x)+\mathrm{N}_{t}} \\
& \times\left(\frac{\bar{\gamma}_{2}}{C \bar{\gamma}_{1} k}\right)^{\frac{1}{2}(-1+l-x)} K_{1-l+x}\left(2 \sqrt{\frac{C \gamma_{\mathrm{th}} k}{\bar{\gamma}_{1} \bar{\gamma}_{2}}}\right)
\end{aligned}
$$

Therefore, $I_{2}$ is achieved by inserting (18) into (15). Finally, substituting (9) and (15) into (8), a closed-form expression for the outage probability of MRNs with opportunistic scheduling and employing beamforming techniques is attained.

\section{NumericAl Results AND Discussions}

In this part, based on the mathematical analysis presented in Section III, some numerical examples are presented in order to evaluate the outage performance of MRNs with fixedgain AF relaying and opportunistic scheduling. Exhaustive simulations have been carried out and compared with the analytical expressions obtained here. As will be observed, all the cases investigated revealed an excellent agreement between analytical and simulation results. In the plots, as in [12] we have normalized the total distance between the source and the destinations to unity so that $d_{S \mathcal{R}}+d_{\mathcal{R} D}=1$. In addition, the variances of the respective channel vectors have been normalized to unity (i.e., $\mathrm{E}\left[\left\|\mathbf{h}_{1}\right\|_{F}^{2}\right]=1, \mathrm{E}\left[\left\|\mathbf{h}_{2, k}\right\|_{F}^{2}\right]=1$ ) and we set $\eta=4$. Moreover, equal average energies are assumed at the source and the relay, i.e., $E_{S \mathcal{R}}=E_{\mathcal{R} D}$. Therefore, the first-hop and the second-hop average SNR attenuates by $d_{S \mathcal{R}}^{-4} E_{S \mathcal{R}} / N_{0}$ and $\left(1-d_{S \mathcal{R}}\right)^{-4} E_{S \mathcal{R}} / N_{0}$.

Fig. 2 depicts the OP against $E_{S \mathcal{R}} / N_{0}$ of MRNs with fixedgain $\mathrm{AF}$ relaying by setting $d_{S \mathcal{R}}=0.3$ and $d_{\mathcal{R} D}=0.7$, and assuming $\mathrm{N}_{t}=\mathrm{N}_{r}$. As can be observed, a slight increase of the number of antennas at the source and destination nodes implies a substantial improvement in performance. This demonstrates the usefulness of the proposed scheme. Also, as expected, an enhancement of the end-to-end performance is noticed as $\gamma_{\text {th }}$ decreases.

In order to to investigate the impact of the relay placement on the outage performance, Fig. 3 plots the OP against $d_{S \mathcal{R}}$ for a threshold value $\gamma_{\mathrm{th}}=0 \mathrm{~dB}$, different values of $L$, and by setting $\mathrm{N}_{t}=\mathrm{N}_{r}$. Herein, we consider $E_{S \mathcal{R}} / N_{0}=10 \mathrm{~dB}$. Interestingly, by keeping $L$ constant, note that as the number of antennas increases, the optimal relay location moves toward to the source. For instance, when $L=3, \mathrm{~N}_{t}=\mathrm{N}_{r}=1$, the optimal relay location is around 0.5 (i.e., $d_{S \mathcal{R}} \approx 0.5$ ), whereas when $L=3, \mathrm{~N}_{t}=\mathrm{N}_{r}=3$, the optimal relay location is around 0.4 (i.e., $d_{S \mathcal{R}} \approx 0.4$ ). One possible justification for this behavior arises from the fact that, when the number of antennas increases, the average symbol energy per-antenna decreases. Then, the relay should be placed closer to the source in order to provide the best system performance once, in the second hop, an opportunistic scheduling will be performed and therefore the probability of finding a good channel is higher than in the first hop. In other words, the first hop becomes the bottleneck for the dual-hop MRNs, since the performance gain achieved from the second hop is significantly higher than the first hop due to the application of opportunistic scheduling.

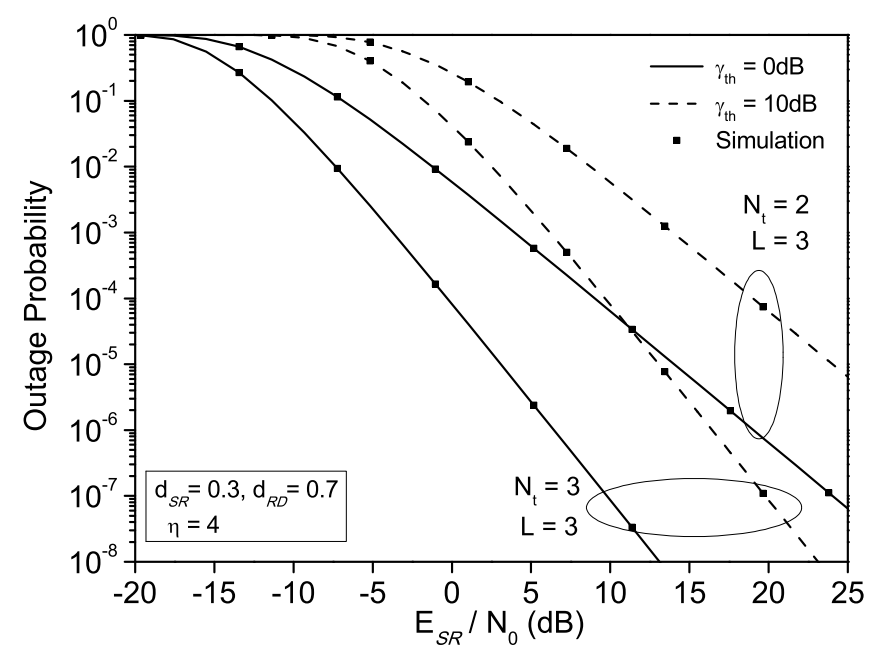

Fig. 2. Outage probability of MRNs with fixed-gain AF relaying for different threshold values and number of antennas $\left(d_{S \mathcal{R}}=0.3, d_{\mathcal{R} D}=0.7, \mathrm{~N}_{t}=\right.$ $\mathrm{N}_{r}$ ).

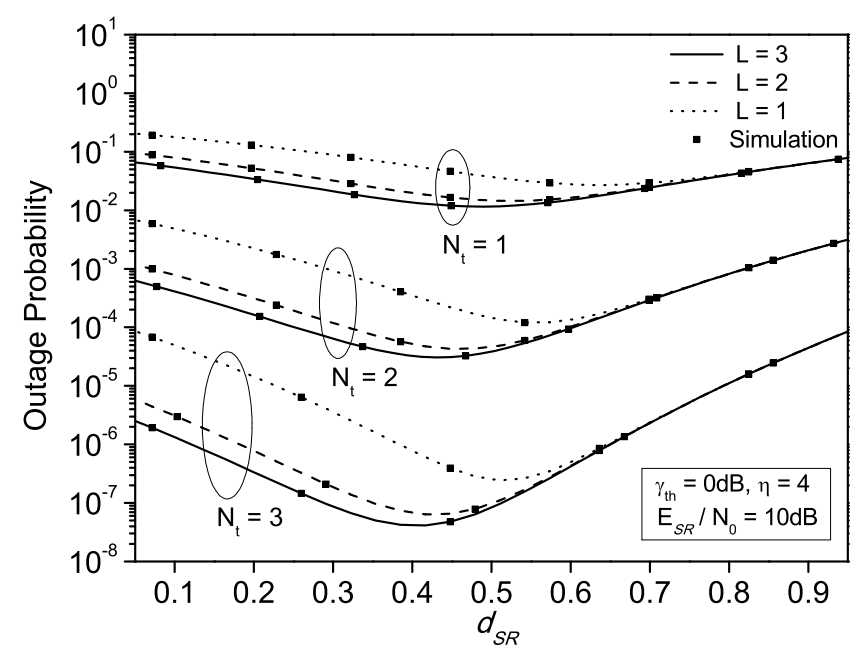

Fig. 3. Outage probability against $d_{S \mathcal{R}}$ of MRNs with fixed-gain AF relaying for different number of antennas and destinations $\left(\gamma_{\mathrm{th}}=0 \mathrm{~dB}, E_{S \mathcal{R}} / N_{0}=\right.$ $10 \mathrm{~dB}, \mathrm{~N}_{t}=\mathrm{N}_{r}$.

\section{CONCLUDING REMARKS}

In this paper, the outage performance of multiuser relay networks was evaluated assuming that the source and each one of the destinations were equipped with multiple antennas and employed beamforming techniques. Our analysis took into account the benefits conferred by opportunistic scheduling in relay-assisted networks. Considering Rayleigh fading and a fixed-gain relay, a closed-form expression for the outage probability was derived. The effects of the relay placement and antenna configurations, on the overall system performance, were discussed through some representative numerical results and comparisons.

For future works, the authors intend to investigate, among 
other things, the following research topics:

- In beamforming systems, perfect channel state information (CSI) is required for a successful transmission. Such knowledge is provided by a feedback channel from the receiver to the transmitter, which usually involves delay. As a result, in practice, the available CSI at the transmitter and the actual channel may be different so that the outdated CSI for beamforming degrades the system performance. The impact of the feedback delay on the end-to-end performance of the considered system is one of the topics that will be studied by the authors later.

- Antenna correlation occurs in many practical scenarios including mobile phones and laptops where the antennas are close together due to space limitations. Thus, the investigation of antenna correlation at the source and destination nodes of the considered system is another interesting open problem.

- It is well-known that Nakagami- $m$ fading covers a wide range of fading scenarios that are typical in realistic wireless applications via the parameter $m$, which comprises the Rayleigh fading $(m=1)$ as a special case. Then, the generalization of the proposed analysis for more flexible fading scenarios is another promising research topic.

- The benefits of relay selection for the system performance is out of question. Then, the inclusion of more relays together with the use of an appropriate relay selection strategy in the proposed analysis emerge as a potential future work.

- The rapid and thriving expansion of wireless systems along the last years, with the demand for new services growing in an incredible pace, has propelled the urge for an efficient use of the radio spectrum. In this sense, frequency reuse has certainly proved to be an effective and practical strategy. However, frequency reuse gives rise to the so-called co-channel interference. Finally, the impact of co-channel interference on the performance is definitely another potential research topic.

\section{REFERÊNCIAS}

[1] P. A. Anghel and M. Kaveh, "Exact symbol error probability of a cooperative network in a Rayleigh-fading environment," IEEE Trans. Wireless Commun., vol. 3, no. 5, pp. 1416-1421, Sep. 2004.

[2] A. Sendonaris, E. Erkip, and B. Aazhang, "User cooperation diversity part I: system description," IEEE Trans. Commun., vol. 51, no. 11, pp. 1927-1938, Nov. 2003.

[3] T. A. Tsiftis, G. K. Karaginnidis, S. A. Kotsopoulus, and F.-N. Pavlidou, "BER analysis of collaborative dual-hop wireless transmission," Electron. Lett., vol. 40, no. 11, pp. 679-680, May 2004.

[4] J. N. Laneman, D. N. C. Tse, and G. W. Wornell, "Cooperative diversity in wireless networks: efficient protocols and outage behavior," IEEE Trans. Inf. Theory, vol. 50, no. 12, pp. 3062-3080, Dec. 2004.

[5] D. Piazza and L. B. Milstein, "Analysis of multiuser diversity in timevarying channels," IEEE Trans. Wireless Commun.,, vol. 6, no. 12, pp. 4412-4419, Dec. 2007.

[6] C.-J. Chen and L.-C. Wang, "A unified capacity analysis for wireless systems with joint multiuser scheduling and antenna diversity in Nakagami fading channels," IEEE Trans. Commun., vol. 54, no. 3, pp. 469-478, Mar. 2006.

[7] Y. Zhao, R. Adve, and T. J. Lim, "Symbol error rate of selection amplifyand-forward relay systems," IEEE Commun. Lett., vol. 10, no. 11, pp. 757-759, Nov. 2006.

[8] — "Improving amplify-and-forward relay networks: optimal power allocation versus selection," IEEE Trans. Wirel. Commun., vol. 6, no. 8, pp. 3114-3123, Aug. 2007.

[9] Z. Yi and I.-M. Kim, "Decode-and-forward cooperative networks with multiuser diversity,” Proc. IEEE MILCOM, pp. 1-7, Oct. 2006.
[10] X. Liu, E. Chong, and N. Shroff, "A framework for opportunistic scheduling in wireless networks," Computer Netw., vol. 41, pp. 451474, Mar. 2003.

[11] L. Yang and M.-S. Alouini, "Performance analysis of multiuser selection diversity," IEEE Trans. Veh. Technol., vol. 55, pp. 1848-1861, Nov. 2006.

[12] N. Yang, M. Elkashlan, and J. Yuan, "Impact of opportunistic scheduling in cooperative dual-hop relay networks," IEEE Trans. Commun., vol. 59, no. 3, pp. 689-694, Mar. 2011.

[13] D. B. da Costa and S. A Aïssa, "Cooperative dual-hop relaying systems with beamforming over Nakagami-m fading channels," IEEE Trans. Wireless Commun., vol. 8, no. 8, pp. 3950-3954, Aug. 2009.

[14] I. S. Gradshteyn and I. M. Ryzhik, Table of Integrals, Series, and Products, 7th ed., San Diego, CA: Academic, 2007.

[15] M. Abramowitz and I. A. Stegun, Handbook of Mathematical Functions with Formulas, Graphs, and Mathematical Tables. New York, 1972.

[16] T. K. Y. Lo, "Maximum ratio transmission," IEEE Trans. Commun., vol. 47, no. 10, pp. 1458-1461, Oct. 1999.

[17] M. O. Hasna and M.-S. Alouini, "A performance study of dual-hop transmissions with fixed gain relays," IEEE Trans. Wireless Commun., vol. 3, no. 6, pp. 1963-1968, Nov. 2004.

[18] T. Tang and R.W. Heath, "Opportunistic feedback for downlink multiuser diversity," IEEE Commun. Lett., vol. 9, no. 10, pp. 948-950, Oct. 2005.

\section{APPENDIX}

Herein, the proof of (12) is detailed. Making use of the binomial theorem [14, Eq. (1.111)], $\Xi$ can be reexpressed as

$$
\Xi=\sum_{k=0}^{L}\left(\begin{array}{l}
L \\
k
\end{array}\right)(-1)^{k}\left[\frac{\Gamma\left(\mathrm{N}_{r}, \frac{C \gamma_{\mathrm{th}}}{\bar{\gamma}_{2}\left(\gamma_{1}-\gamma_{\mathrm{th}}\right)}\right)}{\Gamma\left(\mathrm{N}_{r}\right)}\right]^{k} \text {. }
$$

The incomplete Gamma function presented in (19) can be rewritten in terms of finite sums by means of [14, Eq. (8.352.2)]. Then, it follows that

$$
\left[\frac{\Gamma\left(\mathrm{N}_{r}, \frac{C \gamma_{\mathrm{th}}}{\bar{\gamma}_{2}\left(\gamma_{1}-\gamma_{\mathrm{th}}\right)}\right)}{\Gamma\left(\mathrm{N}_{r}\right)}\right]^{k}=\exp (-\varphi k)\left[\sum_{l=0}^{\mathrm{N}_{r}-1} \frac{1}{l !} \varphi^{l}\right]^{k},
$$

where $\varphi=C \gamma_{\text {th }} /\left(\bar{\gamma}_{2}\left(\gamma_{1}-\gamma_{\text {th }}\right)\right)$. Now, let $\alpha_{l}=1 / l$ !. Noting that the finite series of (20) corresponds to a polynomial $\mathrm{P}$ in $\varphi$ with degree $\left(\mathrm{N}_{r}-1\right)$ and coefficients $\alpha_{l}$, and resorting to the polynomial theory, the $k$-th power of $\mathrm{P}$ can be shown to result in another polynomial with degree $k\left(\mathrm{~N}_{r}-1\right)$ according to

$$
\left[\sum_{l=0}^{\mathrm{N}_{r}-1} \alpha_{l} \varphi^{l}\right]^{k}=\sum_{l=0}^{k\left(\mathrm{~N}_{r}-1\right)} \beta_{l}^{k} \varphi^{l}
$$

whereby using [14, Eq. (0.314)], the coefficients $\beta_{l}^{k}$ can be expressed as

$$
\begin{gathered}
\beta_{0}^{k}=\alpha_{0}^{k}, \beta_{1}^{k}=k \alpha_{1}, \beta_{k\left(\mathrm{~N}_{r}-1\right)}^{k}=\alpha_{\mathrm{N}_{r}-1}^{k} \\
\beta_{l}^{k}=\frac{1}{l \alpha_{0}} \sum_{v=1}^{l}\left[(v k-l+v) \alpha_{v} \beta_{l-v}^{k}\right], 2 \leq l \leq \mathrm{N}_{r}-1 \\
\beta_{l}^{k}=\frac{1}{l \alpha_{0}} \sum_{v=1}^{\mathrm{N}_{r}-1}[(22 \mathrm{~b})
\end{gathered}
$$

Finally, by substituting (20) into (19) with the $k$-th power of the finite series pertaining to the former rewritten as (21), a closed-form expression for $\Xi$ can be obtained as shown in (12). In this case, (13) is obtained from (22a) considering $\alpha_{l}=1 / l$ !, while (14) is obtained by grouping (22b) and (22c). 